\title{
Fractura del tubérculo medial de la apófisis posterior del astrágalo
}

\section{Medial Tubercle's Fracture of Posterior Process of Talus}

\author{
María Tíscar García-Ortiz ${ }^{1}$ [ Lorena Moril-Peñalver $^{1} \quad$ Alexandra Sevilla-Monllor $^{1}$ \\ María Dolores Fernández-Ruiz ${ }^{1}$ Issam Menchi-Elanzi ${ }^{1}$ Fernando López-Prats ${ }^{1,2}$ (D) \\ ${ }^{1}$ Departamento de Cirugía Ortopédica y Traumatología del Hospital \\ General Universitario de Elche, Elche, Alicante, España \\ 2 Departamento de Cirugía Ortopédica y Traumatología, Universidad \\ Miguel Hernández, San Juan de Alicante, España \\ Address for correspondence María Tíscar García Ortiz, MD, Calle \\ Palangre número 21. Bajo CP:03195., El Altet, Alicante, España \\ (e-mail: tiscargarciaortiz@gmail.com). \\ Rev Chil Ortop Traumatol 2020;61:94-100.
}

\section{Resumen}

Palabras clave

- fractura de cedell

- fractura del tubérculo medial de la apófisis posterior del astrágalo

\section{Abstract}

Objetivo Mostrar una fractura infrecuente del tubérculo posteromedial del astrágalo diagnosticado y tratado de manera aguda mediante osteosíntesis con tornillo Acutrak $\circledast$ (2014 Acumed $\circledast$ LLC).

Material y Métodos Varón de 28 años, que acude a Urgencias tras torcedura de tobillo con dolor al mover tobillo y hallux y tumefacción en cara interna. En Urgencias pasó desapercibida. En consulta a la semana refería sensación de que se le engancha el primer dedo con la flexoextensión. Se observa fractura del tubérculo medial de la apófisis posterior del astrágalo con desplazamiento $>3 \mathrm{~mm}$ y clínica de posible interposición del flexor hallucis longus. Se decidió tratamiento quirúrgico con tornillo Acutrak®. Se inmovilizó con férula 3 semanas y descarga 6 semanas.

Resultados A los 8 meses balance articular completo, sin dolor ni limitación para las ABVD y sin clínica de atrapamiento del flexor del hallux. Como complicación aguda, se verificó infección de herida quirúrgica que se trató con antibióticos.

Discusión Ese tipo de fracturas son infrecuentes. El mecanismo lesional suele ser dorsiflexión-pronación. Es importante un diagnóstico temprano y para ello es necesario una alta sospecha diagnóstica. Para su diagnóstico, son necesarias radiografías anteroposterior y lateral y si no se visualiza la lesión, proyección oblicua con $30^{\circ}-40^{\circ}$ de rotación externa. En casos agudos resulta de utilidad la TAC y en casos crónicos la RM.

Conclusión Aunque generalmente el tratamiento es conservador, en ocasiones puede ser necesario la cirugía mediante osteosíntesis o exéresis del fragmento cuando la fractura provoca impingement.

Objective Show an infrequent fracture of the posteromedial tubercle of the talus diagnosed and treated acutely by Acutrak ${ }^{\circledR}$ screw (2014 Acumed $\circledast$ LLC) osteosynthesis. Material and Methods A 28-year-old man attended the Emergency Department after spraining his ankle with pain when moving his ankle and hallux and swelling on the internal face. In the ER it went unnoticed. A week later in the clinic, he refered to the sensation that the first finger is hooked with the flexion extension. A fracture of the received

April 25, 2020

accepted

June 29, 2020
DOI https://doi.org/

$10.1055 / \mathrm{s}-0040-1715492$.

ISSN $0716-4548$
Copyright $\odot 2020$ by Thieme Revinter

Publicações Ltda, Rio de Janeiro, Brazil
License terms

()ㅇㅇ $\Theta \circledast$ 


\section{Keywords}

- cedell's fracture

- medial tubercle's fracture of posterior process of talus medial tubercle of the posterior process of the talus is observed with a displacement of $>3 \mathrm{~mm}$ and clinical signs of possible interposition of the hallucis longus . Surgical treatment with Acutrak ${ }^{\circ}$ screw was made. He was immobilized with a splint for 3 weeks and discharge for 6 weeks.

Results At 8 months complete joint balance, without pain or limitations for BADL and without hallux flexor entrapment symptoms. As an acute complication, surgical wound infection that was treated with antibiotics.

Discussion These types of fractures are rare. The injury mechanism is usually dorsiflexion-pronation. Early diagnosis is important and a high diagnostic suspicion is required. Anteroposterior and lateral radiographs are necessary for its diagnosis and if the lesion is not visualized, oblique projection with $30^{\circ}-40^{\circ}$ external rotation. CT is useful in acute cases and MRI in chronic cases.

Conclusion Although the treatment is conservative, surgery may sometimes be necessary by means of osteosynthesis or excision of the fragment when the fracture causes impingement.

\section{Introducción}

Las fracturas del tubérculo medial de la apófisis posterior del astrágalo son infrecuentes. Descrita en 1974 por Cedell, su mecanismo lesional es una combinación de dorsiflexión y pronación, ${ }^{1-3}$ aunque también se han descrito por traumatismo directo ${ }^{4}$, impingement del sustentaculum tali en supinación ${ }^{5}$ o dorsiflexión forzada en traumatismo de alta energía. ${ }^{6,7}$

Recordando la anatomía ( - Figura 1), el proceso posterior del astrágalo consta de dos tubérculos, uno lateral y otro medial. Surge de un centro de osificación secundario que se fusiona con el cuerpo del astrágalo alrededor de los 12 años de edad. El tubérculo postero-lateral es el más grande de los dos y proporciona una unión para el ligamento talofibular posterior, mientras que el tubérculo postero-medial es más pequeño y actúa como el punto de inserción para los componentes talotibiales del ligamento deltoideo (el tercio posterior) $)^{8-10}$ y la parte medial de la bifurcación del ligamento astrágalo-calcáneo inferiormente. ${ }^{11-13}$
Es importante realizar un correcto diagnóstico de la fractura del tubérculo medial de la apófisis posterior del astrágalo y para ello es preciso tener una alta sospecha clínica, localizándose el dolor inmediatamente inferior al margen posterior del maleolo interno, debido a que en muchas ocasiones pasa desapercibida y puede ser diagnosticada como esguince de tobillo, síndrome de os trigonum, bursitis retrocalcánea, tendinopatía del tibial posterior o tendinopatía del flexor hallucis longus. ${ }^{14-19}$ Por lo tanto, de entrada sería necesario una radiografía anteroposterior y lateral y si no se visualiza la lesión añadir una radiografía oblicua con $30^{\circ}-40^{\circ}$ de rotación externa. ${ }^{14,20}$

Para su diagnóstico en casos agudos resulta de utilidad la $\mathrm{TAC}^{21,22}$ y en casos crónicos la RM. ${ }^{23,24}$ Ese tipo de lesiones tiene una importante morbilidad si pasan desapercibidas generando inestabilidad y dolor crónico a nivel del tobillo. ${ }^{25-27}$

Generalmente el tratamiento conservador suele ser el tratamiento de elección mediante inmovilización con férula suropédica o yeso cerrado durante 6 semanas. El tratamiento quirúrgico se reserva para desplazamiento $\geq 3 \mathrm{~mm}$, compresión

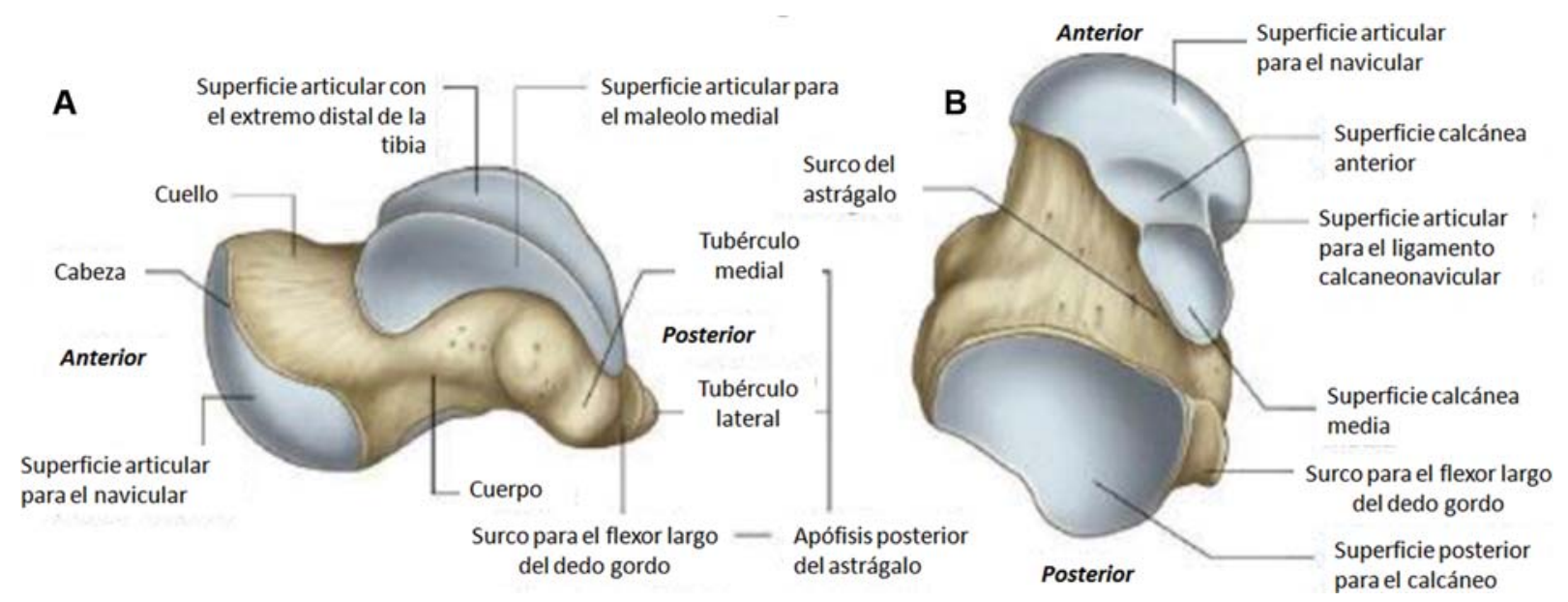

Fig. 1 Anatomía del astrágalo. ${ }^{11}$ 
Tabla 1 En el mecanismo de lesión de las fracturas del tubérculo medial de la apófisis posterior del astrágalo, el ligamento deltoideo posterior avulsiona el tubérculo medial de la parte posterior mediante un mecanismo de dorsiflexión y pronación. . $^{1,8,38}$

\begin{tabular}{|c|c|c|c|c|}
\hline Tipo de fractura & $\begin{array}{l}\text { Mecanismo } \\
\text { lesional }\end{array}$ & Hallazgos clínicos & $\begin{array}{l}\text { Mejor proyección } \\
\text { radiológica }\end{array}$ & Tratamiento \\
\hline $\begin{array}{l}\text { Apófisis posterior } \\
\text { del astrágalo } \\
\text { (Tubérculo medial). }\end{array}$ & $\begin{array}{l}\text { Dorsiflexión } \\
\text { y pronación. }\end{array}$ & $\begin{array}{l}\text { Dolor a la palpación entre el } \\
\text { maleolo interno y el tendón de } \\
\text { Aquiles. }\end{array}$ & $\begin{array}{l}\text { Radiografía oblicua } \\
\text { del tobilllo con el pie } \\
\text { a } 40^{\circ} \text { de rotación } \\
\text { externa. }\end{array}$ & \multirow{3}{*}{$\begin{array}{l}\text { Sin desplazar: Inmovilización con } \\
\text { férula 4-6 semanas. } \\
\text { Desplazadas } \geq 3 \mathrm{~mm} \text { : Plantear } \\
\text { tratamiento quirúrgico. }\end{array}$} \\
\hline $\begin{array}{l}\text { Apófisis posterior } \\
\text { del astrágalo } \\
\text { (Tubérculo lateral). }\end{array}$ & $\begin{array}{l}\text { Hiperflexión } \\
\text { plantar o } \\
\text { inversión } \\
\text { forzada. }\end{array}$ & $\begin{array}{l}\text { Dolor a la palpación } \\
\text { anterior al tendón de Aquiles } \\
\text { sobre la cara posterolateral del } \\
\text { astrágalo y dolor con la flexión } \\
\text { plantar. }\end{array}$ & Radiografía lateral. & \\
\hline $\begin{array}{l}\text { Apófisis lateral del } \\
\text { astrágalo. }\end{array}$ & $\begin{array}{l}\text { Inversión y } \\
\text { dorsiflexión. }\end{array}$ & $\begin{array}{l}\text { Dolor anterior e inferior al } \\
\text { maleolo externo. }\end{array}$ & $\begin{array}{l}\text { Radiografía lateral y } \\
\text { proyección de la } \\
\text { mortaja. }\end{array}$ & \\
\hline
\end{tabular}

del paquete vasculonervioso tibial posterior ${ }^{28}$, interposición de flexor del hallucis longus ${ }^{6,29,30}$ o afectación articular. ${ }^{31,32}$ Las diferentes opciones quirúrgicas van desde la osteosíntesis con tornillos de pequeños fragmentos o mini ${ }^{33}$, tornillos de Herbert, $^{18}$ la fijación con agujas Kirschner, ${ }^{25}$ fijación con pines bioabsorbibles ${ }^{34}$ o la exéresis del fragmento. ${ }^{35} \mathrm{El}$ abordaje para la fijación suele ser posteromedial. ${ }^{36}$ En caso de diagnóstico tardío se puede realizar exéresis del fragmento bien con cirugía abierta o mediante artroscopia. ${ }^{37}$

El error diagnóstico genera desplazamiento de las fracturas no desplazadas y en ocasiones se tratan más tarde las secuelas; como el dolor, la pseudoartrosis, la interposición del flexor hallucis longus o el síndrome del túnel del tarso, ${ }^{18}$ que en numerosas ocasiones no se presentarían con un diagnóstico precoz y el tratamiento correspondiente. $^{38}$ - Tabla 1

\section{Material y Métodos}

Varón de 28 años sin antecedentes de interés que acudió a Urgencias tras torcedura de tobillo izquierdo. A la exploración presentaba dolor y tumefacción en cara interna del tobillo, además de dolor con la movilización del tobillo y del hallux. Exploración neurovascular distal normal. Se le realizó una

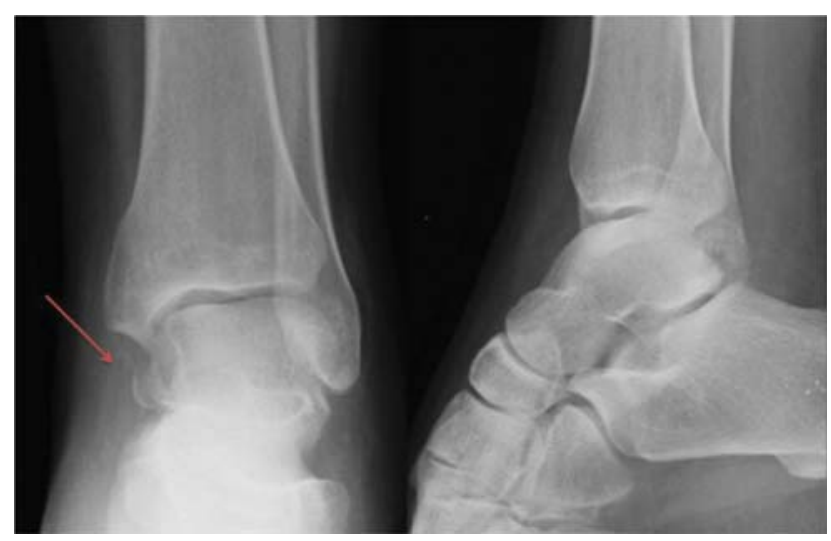

Fig. 2 Radiología simple realizada en Urgencias que muestra fractura de tubérculo medial de la apófisis posterior astrágalo, que en un primer momento pasó desapercibida. radiografía AP y lateral del tobillo izquierdo donde fue diagnosticado de esguince (-Figura 2). Se le colocó una férula posterior suropédica y se derivó a consultas externas. En el primer control a los 7 días de la lesión inicial, el paciente refiere sensación de que se le engancha el primer dedo con el movimiento de flexoextensión.

En el control en consultas externas se observa una fractura del tubérculo medial de la apófisis posterior del astrágalo. Se completó el estudio mediante TAC (-Figura 3) en el que se observa fractura de la apófisis posterior del astrágalo y una fractura de la apófisis anterior del calcáneo (- Figura 4).

En nuestro caso puesto que se trataba de una lesión aguda intraarticular, con un desplazamiento mayor de $3 \mathrm{~mm}$ y clínica de posible interposición del flexor hallucis longus, se decidió el tratamiento quirúrgico.

\section{Resultados}

Se realizó una osteosíntesis con un tornillo Acutrak® de pequeños fragmentos mediante un abordaje medial referenciando anteriormente el tendón flexor hallucis longus y posteriormente el paquete vasculonervioso tibial posterior (-Figuras 5-9).

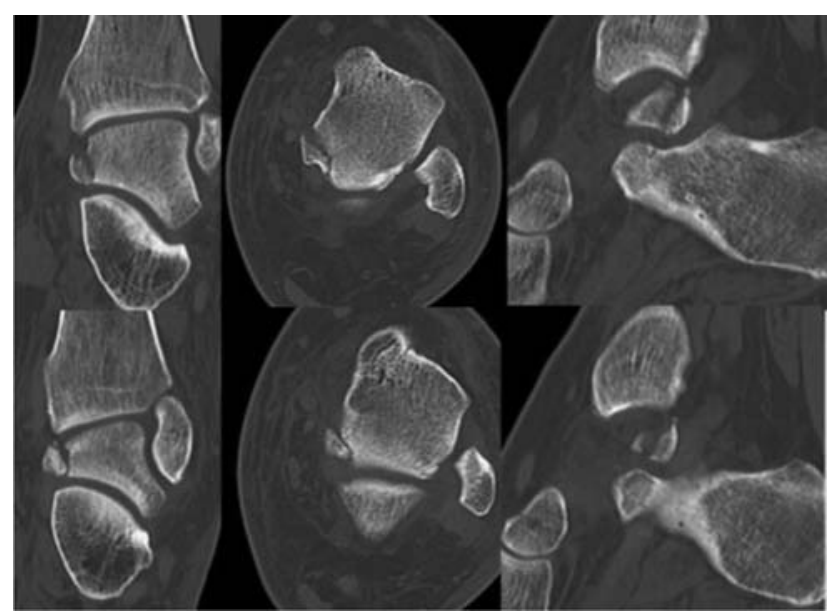

Fig. 3 TAC tobillo izquierdo, Fractura del tubérculo medial de la apófisis posterior del astrágalo. 


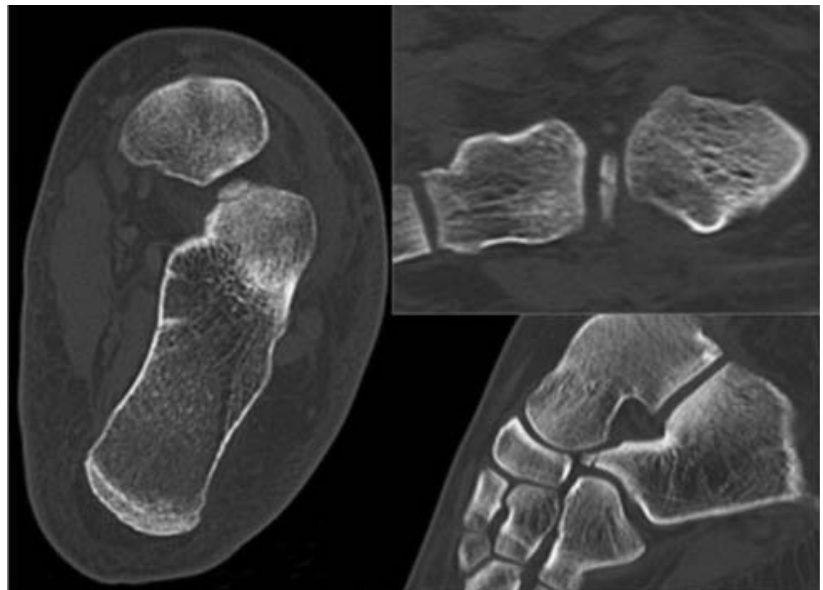

Fig. 4 TAC. Fractura de la apófisis anterior del calcáneo.

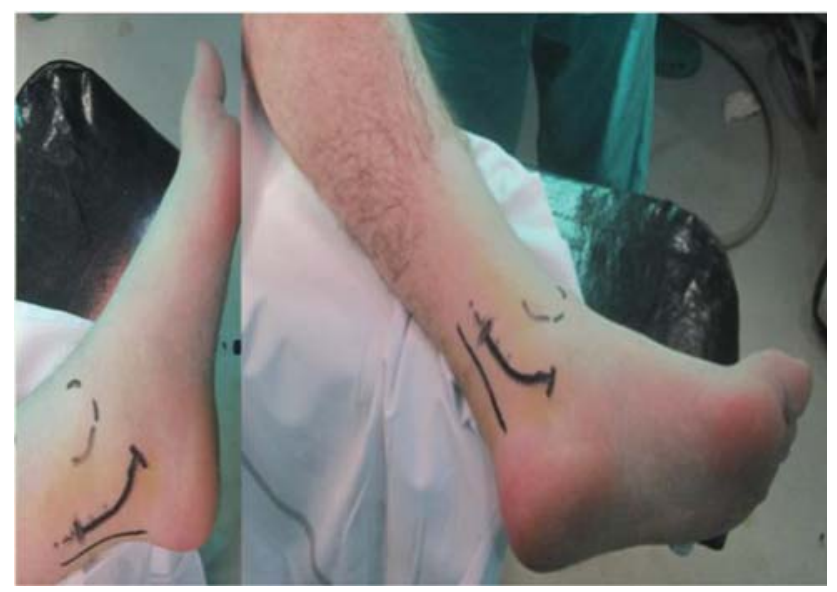

Fig. 5 Vía de abordaje.

Se inmovilizó con férula suropédica durante 3 semanas y posteriormente se inició movilización activa del tobillo. Se mantuvo en descarga absoluta durante 6 semanas, luego dos semanas con carga parcial y a la octava semana se remitió a RHB y se inició carga completa. Como complicación, el

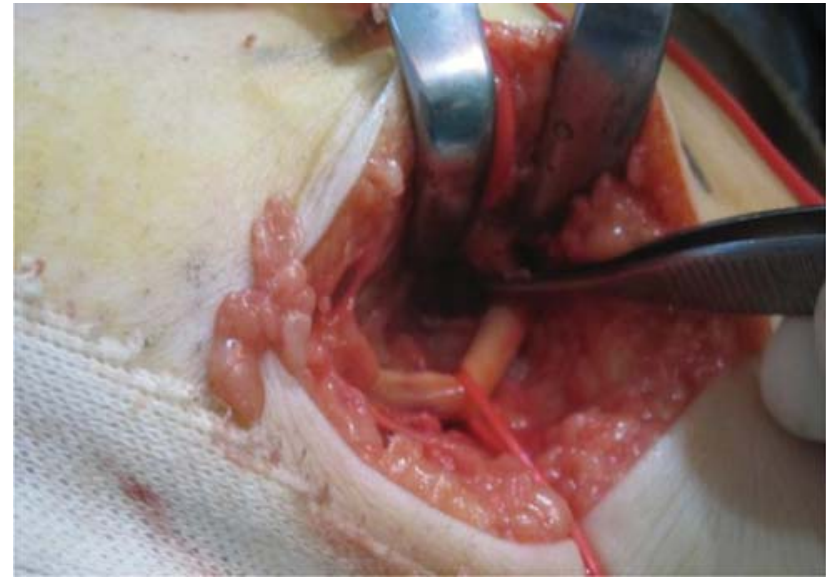

Fig. 6 Disección paquete Vasculonervioso tibial posterior.

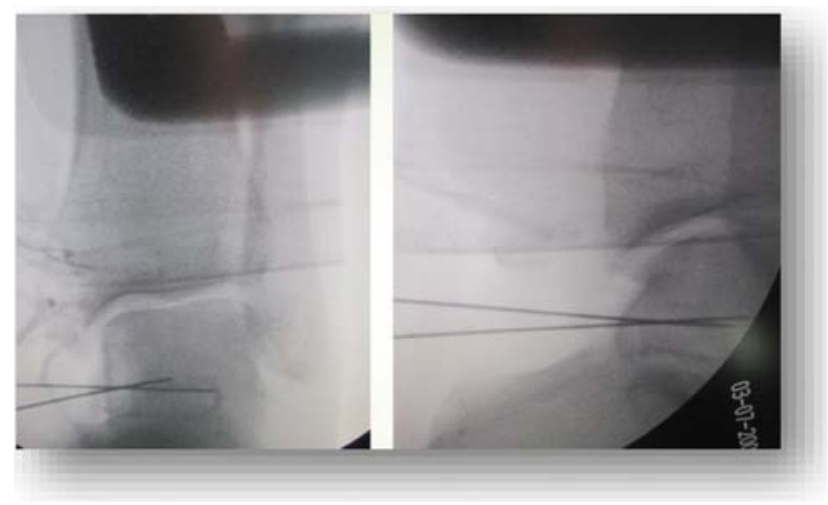

Fig. 7 Reducción del fragmento con agujas Kirschner.

paciente tuvo una infección superficial de la herida quirúrgica con dehiscencia de un punto en la zona central de la cicatriz que se trató con antibioterapia empírica con Amoxicilina-Clavulánico durante una semana hasta que se tuvo el resultado del cultivo en el cual se aisló Acinetobacter

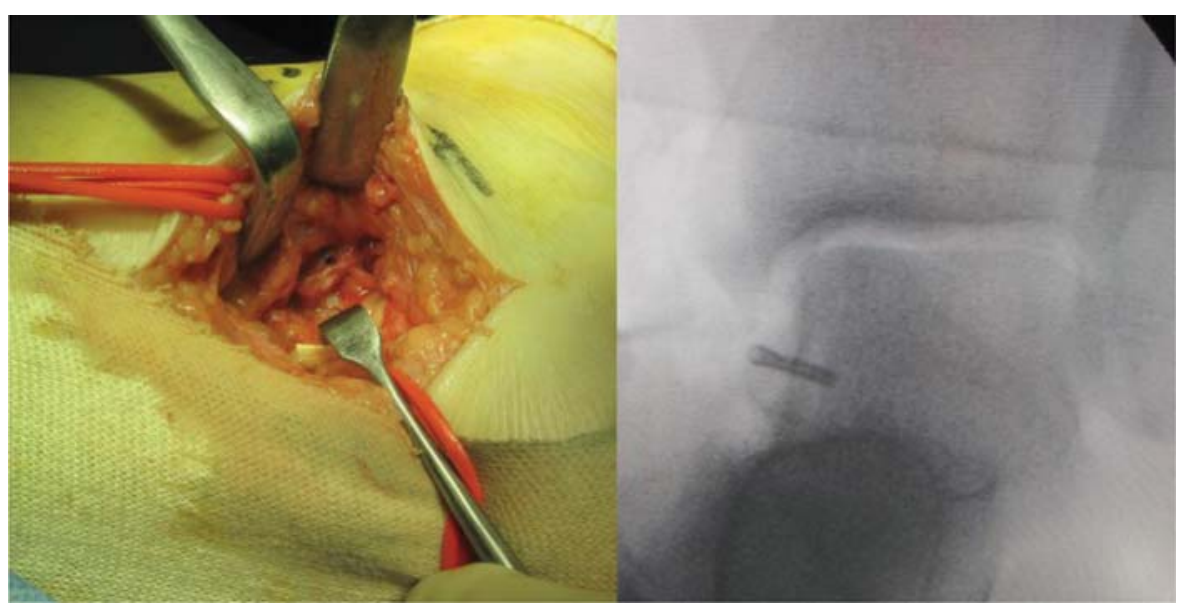

Fig. 8 Osteosíntesis con tornillo Acutrak $®$ de pequeños fragmentos. a) Visión clínica b) Visión radiológica. 


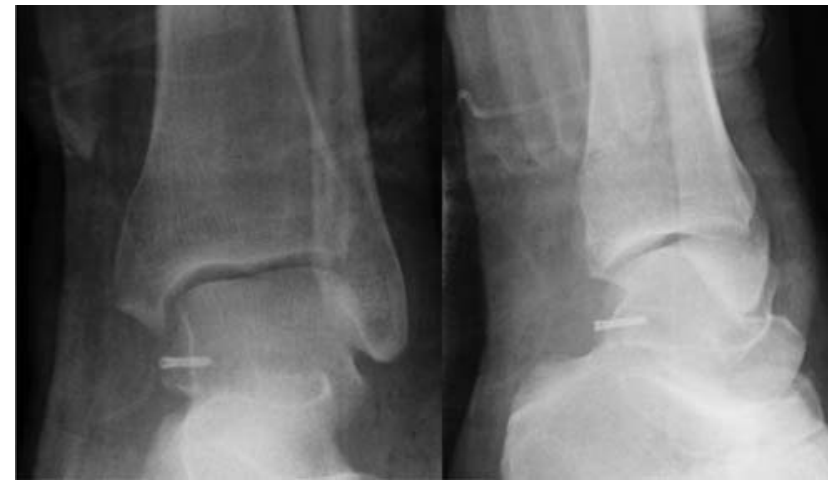

Fig. 9 Control postquirúrgico. que definió la causa como la combinación de dorsiflexión y pronación en pacientes deportistas, en los cuales el ligamento astrágalo-tibial posterior que se extiende desde la parte dorsal y distal del maleolo medial hasta el tubérculo medial del astrágalo, producía una fractura de esa apófisis. Por el contrario, los casos en que la apófisis fracturada es la posterolateral se denominan Fracturas de Shepherd (1882) y son producidas por un mecanismo de flexión plantar e inversión. Es importante no confundir este tipo de lesiones con el Os Trigonum, hueso sesamoideo presente casi en el $50 \%$ de los pies y que suele ser bilateral. Para el correcto diagnóstico de este tipo de lesiones, es importantísimo la anamnesis, exploración física y radiología simple (AP lateral y oblicua con $30^{\circ}-40^{\circ}$ de rotación externa). Además el uso de

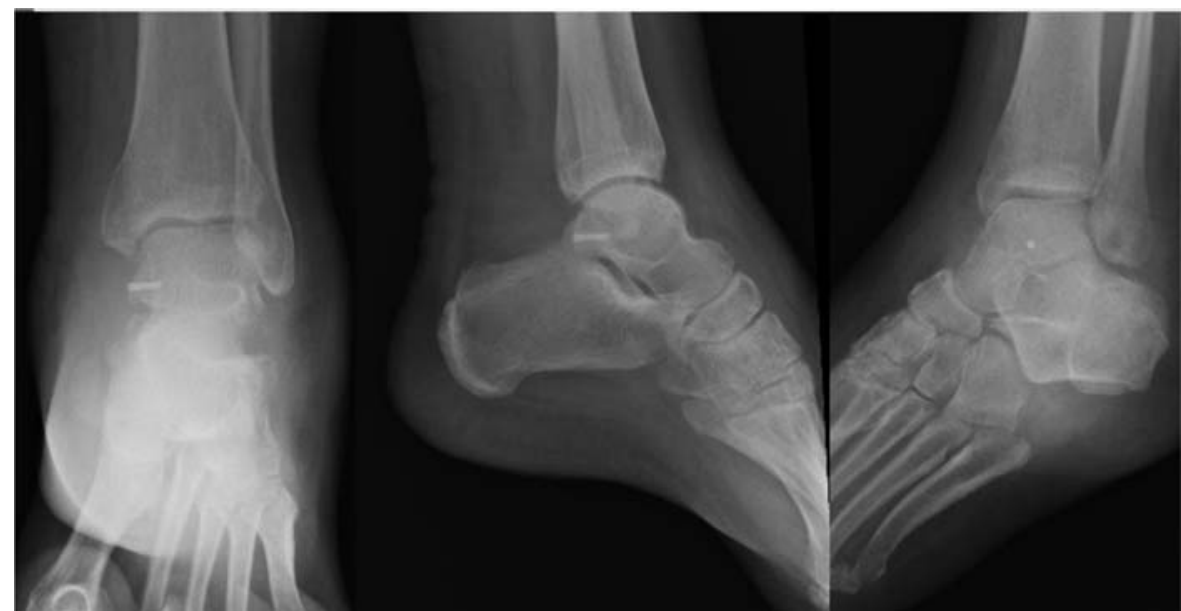

Fig. 10 Control 2,5 meses postquirúrgicos.

Baumanni haemolyticus sensible a Ciprofloxacino. Se pautó Ciprofloxacino $750 \mathrm{mg} / 12$ horas una semana y 500mg /12h dos semanas más. La evolución de la infección fue favorable y se resolvió el proceso de cicatrización.

A los 2,5 meses presenta un balance articular completo, sin dolor ni limitación para las ABVD y sin clínica de atrapamiento del flexor del hallux. (-Figura 10). En la última revisión a los 8 meses el paciente se encuentra clínicamente bien.

\section{Discusión}

Las fracturas del astrágalo son las segundas en frecuencia (después del calcáneo) de todas las fracturas del tarso. Anatómicamente podemos dividirlo en diferentes zonas: cuerpo, cuello, cabeza, procesos posterior y lateral y seno del tarso. Por lo que respecta al proceso lateral, forma parte de la carilla articular y se articula con el peroné y el calcáneo. Por otro lado, el proceso posterior o apófisis posterior, está formada por dos tubérculos, uno medial y otro lateral, separados entre sí por un surco para el flexor del hallux. La fractura del tubérculo medial de la apófisis posterior del astrágalo fue por primera vez descrita en 1974 por Cedell, la RMN, la TAC y el SPECT-TAC, ${ }^{39}$ pueden resultar muy útiles para el diagnóstico y ayudarnos a tomar la decisión terapéutica oportuna. Generalmente el tratamiento es conservador con yeso sin carga entre 4-6 semanas, pero en ocasiones puede ser preciso el tratamiento quirúrgico mediante osteosíntesis o exéresis del fragmento ${ }^{40}$ en casos no osteosintetizables.

Nuestro paciente permaneció 3 semanas con férula y otras 3 semanas en descarga tras la fijación interna. A los 2,5 meses, la carga completa no producía dolor ni limitación para las ABVD, aproximadamente el tiempo de recuperación habitual cuando se realiza un diagnóstico precoz. El tratamiento quirúrgico suele reservarse para aquellos casos con desplazamientos ${ }^{3}$ $\geq 3 \mathrm{~mm}$, compresión del paquete vasculonervioso tibial posterior $^{28}$, interposición del flexor hallucis longus ${ }^{6,29,30}$ o afectación articular. ${ }^{31,32}$ Es decir, existe un amplio abanico de opciones quirúrgicas que van desde la osteosíntesis con tornillos de pequeños fragmentos o mini ${ }^{33}$, tornillos de Herbert, ${ }^{18}$ la fijación con agujas Kirschner, ${ }^{25}$ fijación con pines bioabsorbibles ${ }^{34}$ o la exéresis del fragmento. ${ }^{35}$ Cuando la fractura provoca un impingement la resección del fragmento es el tratamiento recomendado. ${ }^{37}$ Giuffrida y col., recomiendan la escisión de los pequeños fragmentos de la 
fractura en caso de no afectar la superficie articular subtalar. Mientras que las fracturas que involucran suficiente superficie articular para causar subluxación o inestabilidad se tratan con reducción abierta y fijación interna. ${ }^{41}$ Actualmente, la artroscopia de tobillo es una técnica en auge que ofrece la posibilidad de realizar la exéresis temprana del fragmento en caso de no posibilidad de osteosíntesis ${ }^{42}$ o en casos de pseudoartrosis mediante portales artroscópicos ${ }^{16}$, pero también es una posibilidad terapéutica para la fijación de fragmentos osteocondrales. En la literatura hay recogidos casos de fijación artroscópica en fragmentos osteocondrales o fracturas del proceso lateral del astrágalo ${ }^{42}$, pero sin haber encontrado literatura al respecto sobre la fijación artroscópica de las fracturas del tubérculo posteromedial.

Por último, reiteramos que es primordial remarcar la importancia del diagnóstico precoz de ese tipo de fracturas, ya que en muchas ocasiones pasa desapercibida en los servicios de Urgencias o se confunde con el Os Trigonum, por lo que dada la alta morbilidad que supone para el paciente y las graves secuelas que pueden causar, como la artrosis subastragalina $^{24}$, es vital tener la sospecha diagnóstica para poder realizar un diagnóstico precoz. La poca casuística publicada en la bibliografía, muchas veces en forma de pseudoartrosis, ${ }^{43,44}$ hace de este caso diagnosticado $\mathrm{y}$ tratado de manera precoz, un caso interesante.

\section{Conclusión}

En nuestro paciente, fue clave el tratamiento precoz para una recuperación temprana y completa. Es fundamental para el mantenimiento de la función del miembro, el diagnóstico temprano y para ello es importante tener la sospecha diagnóstica dada la baja incidencia de estas lesiones. En nuestro caso, se utilizó un tornillo de pequeños fragmentos dado el tamaño del tubérculo que resultó en un resultado clínico y funcional excelente.

\section{Conflictos de intereses}

Los autores declaran no tener ningún conflicto de intereses.

\section{Referencias}

1 Cedell CA. Rupture of the posterior talotibial ligament with the avulsion of a bone fragment from the talus. Acta Orthop Scand 1974;45(03):454-461

2 Kinner B, Roll C. Peripheral Talus Fractures-A Clinical Observational Study of 16 Cases. Clin Res Foot Ankle 2018;6:264

$3 \mathrm{Kim}$ DH, Berkowitz MJ, Pressman DN. Avulsion fractures of the medial tubercle of the posterior process of the talus. Foot Ankle Int 2003;24(02):172-175

4 Wolf RS, Heckman JD. Case report: fracture of the posterior medial tubercle of the talus secondary to direct trauma. Foot Ankle Int 1998;19(04):255-258

5 Cohen AP, Conroy JL, Giannoudis PV, Matthews SJ, Smith M. Impingement fracture of the posteromedial process of the talus-a case report. Acta Orthop Scand 2000;71(06):642-644

6 Dougall TW, Ashcroft GP. Flexor hallucis longus tendon interposition in a fracture of the medial tubercle of the posterior process of the talus. Injury 1997;28(08):551-552
7 Watanabe H, Majima T, Takahashi K, Kawaji H, Takai S. Split fracture of the posteromedial tubercle of the talus: Case report and proposed classification system. J Foot Ankle Surg 2017;56(01):187-190

8 Judd DB, Kim DH. Foot fractures frequently misdiagnosed as ankle sprains. Am Fam Physician 2002;66(05):785-794

9 Nasser S, Manoli A II. Fracture of the entire posterior process of the talus: a case report. Foot Ankle 1990;10(04):235-238

10 Heckman JD. Fractures and dislocation of the foot. Fractures in Adults 1996

11 Williams PL. Gray's anatomy. Nervous System 1995:1240-1243

12 Sarrafian SK. Anatomy of the foot and ankle. 1993 2Philadelphia; 1983

13 Utrilla AL, López-Prats F. Consideraciones anatómicas, mecanismos y valoración de resultados en las fracturas de astrágalo. Rev Ortop Traumatol (B Aires) 2007;51:31-40

14 Ebraheim NA, Patil V, Frisch NC, Liu X. Diagnosis of medial tubercle fractures of the talar posterior process using oblique views. Injury 2007;38(11):1313-1317

15 Kim DH, Hrutkay JM, Samson MM. Fracture of the medial tubercle of the posterior process of the talus: a case report and literature review. Foot Ankle Int 1996;17(03):186-188

16 Ebraheim NA, Padanilam TG, Wong FY. Posteromedial process fractures of the talus. Foot Ankle Int 1995;16(11):734-739

17 Kanbe K, Kubota H, Hasegawa A, Udagawa E. Fracture of the posterior medial tubercle of the talus treated by internal fixation: a report of two cases. Foot Ankle Int 1995;16(03):164-166

18 Stefko RM, Lauerman WC, Heckman JD. Tarsal tunnel syndrome caused by an unrecognized fracture of the posterior process of the talus (Cedell fracture). A case report. J Bone Joint Surg Am 1994; 76(01):116-118

19 Majeed H, McBride DJ. Talar process fractures: An overview and update of the literature. EFORT Open Rev 2018;3(03):85-92

20 Ebraheim NA, Karkare N, Gehling DJ, Liu J, Ervin D, Werner CM. Use of a 30-degree external rotation view for posteromedial tubercle fractures of the talus. J Orthop Trauma 2007;21(08):579-582

21 Ebraheim NA, Skie MC, Podeszwa DA, Jackson WT. Evaluation of process fractures of the talus using computed tomography.J Orthop Trauma 1994;8(04):332-337

22 Benmansour MB, Gottin M, Leclercq S, Dintimille H, Dib C, Catonne Y. [Associated fracture of the posteromedial tubercle and the lateral process of the talus: a case report. Contribution of computed tomography]. Rev Chir Orthop Repar Appar Mot 1999; 85(07):744-748

23 O'Loughlin P, Sofka CM, Kennedy JG. Fracture of the medial tubercle of the posterior process of the talus: magnetic resonance imaging appearance with clinical follow-up. HSS J 2009;5(02):161-164

24 Albert P, Patel J, Katz JI, Loria F, Parnell J, Brenner M. Magnetic resonance imaging, computed tomography, and radiographic correlation of nonunion of the posteromedial tubercle of the talus: a case report. J Foot Ankle Surg 2014;53(06):787-790

25 Berkowitz MJ, Kim DH. Process and tubercle fractures of the hindfoot. J Am Acad Orthop Surg 2005;13(08):492-502

26 Paulos LE, Johnson CL, Noyes FR. Posterior compartment fractures of the ankle. A commonly missed athletic injury. Am J Sports Med 1983;11(06):439-443

27 Ahmad R, Ahmed SMY. Fracture of the posterior process of the talus: an unusual injury. Emerg Med J 2007;24(12):867

28 Gómez Robledo J. Associated lateral process and posteromedial tubercle talus fractures with entrapment of the medial neurovascular bundle: a case report. Foot 2013;23(04):149-153

29 Park J, Che JH. Talus Fracture of the Medial Tubercle of the Posterior Process: Interposition of the Flexor Hallucis Longus Tendon. J Foot Ankle Surg 2018;57(03):615-617

30 Herrera-Rodríguez A, Martínez-Romero JL. Fracturas de la cabeza, cuerpo y apófisis del astrágalo. Rev Ortop Traumatol (B Aires) 2007;51:41-50 
31 Bhanot A, Kaushal R, Bhan R, Gupta PN, Gupta RK, Bahadur R. Fracture of the posterior process of talus. Injury 2004;35(12): 1341-1344

32 Hsu AR, Scolaro JA. Posteromedial approach for open reduction and internal fixation of talar process fractures. Foot Ankle Int 2016;37(04):446-452

33 Chen CW, Hsu SY, Wei YS. Fracture of the medial tubercle of the posterior process of the talus. Formosan Journal of Musculoskeletal Disorders 2011;2(02):62-65

34 Letonoff EJ, Najarian CB, Suleiman J. The posteromedial process fracture of the talus: a case report. J Foot Ankle Surg 2002;41(01): 52-56

35 Gutierres M, Cabral T, Miranda A, Almeida L. Fractures of the posteromedial process of the talus. A report of two cases. Int Orthop 1998;22(06):394-396

36 Banks AS, Caldarella D. Fractures of the posteromedial process of the talus. J Am Podiatr Med Assoc 1994;84(02):66-70

37 Más Martínez J, Verdú Román C, Martínez Giménez E, SanzReig J, Bustamante Suárez de Puga D, Morales Santías M. Arthroscopic treatment of a malunion of a posteromedial tubercle fracture of the talus. Arthrosc Tech 2017;6(06): e2107-e2110
38 Komur B, Yilmaz B, Kati YA, Turan A, Faruk O. Fracture of the entire posterior process of the talus: A case report and review of the literature. Int J Orthod 2017;3(01):640-644

39 Olivier D, Florent G, Philippe R, Ronan A, Solène Q. Nonunion of the Posteromedial Tubercle of the Talus: Interest of SPECT/CT. J Nucl Med Radiat Ther 2016;7(299):2

40 Yiannakopoulos CK, Kanellopoulos AD. Avulsion fracture of the medial tubercle of the posterior process of the talus: Threedimensional (3D) computed tomography features. Foot 2007;17 (01):38-41

41 Giuffrida AY, Lin SS, Abidi N, Berberian W, Berkman A, Behrens FF. Pseudo os trigonum sign: missed posteromedial talar facet fracture. Foot Ankle Int 2003;24(08):642-649

42 Hepple S, Guha A. The role of ankle arthroscopy in acute ankle injuries of the athlete. Foot Ankle Clin 2013;18(02):185-194

43 Van Tongel A, Matricali GA. Fracture of the posterior medial tubercle of the talus: a case report and review of the literature. Acta Orthop Belg 2007;73(06):804-806

44 Rogosić S, Bojanić I, Borić I, Tudor A, Srdoc D, Sestan B. Unrecognized fracture of the posteromedial process of the talus-a case report and review of literature. Acta Clin Croat 2010;49(03):315-320 